\title{
Advances in $s$-process models
}

\author{
M. Lugaro ${ }^{\mathrm{a} *}$ and F. Herwig ${ }^{\mathrm{b} \dagger}$

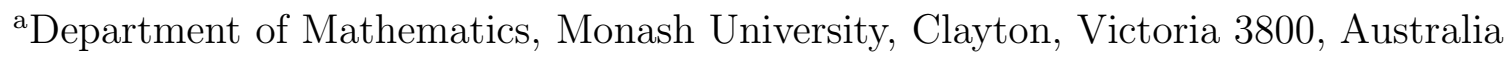 \\ ${ }^{\mathrm{b}}$ Universitaet Potsdam, Institut fuer Physik, Astrophysik, D-14469 Potsdam, Germany
}

Within the framework of the current models for the slow neutron capture $(s)$ process in Asymptotic Giant Branch (AGB) stars we explore the uncertainties introduced by the assumptions made on stellar modeling. On the basis of star models constructed with three different evolutionary codes we generate detailed neutron-capture nucleosynthesis postprocessing models. The main difference among the codes is that one of them includes an overshooting mechanism. As a result, the neutron fluxes are stronger both during the interpulse periods and within thermal pulses. The efficiency of the ${ }^{13} \mathrm{C}$ source is also studied and we find that a linear relationship exists between the initial ${ }^{12} \mathrm{C}$ in the intershell and the maximum number of neutrons produced.

\section{INTRODUCTION}

The $s$ process had been suggested in the 1950s to account for the nucleosynthesis of elements heavier than Fe [1]. The $s$-process path of neutron capture generally follows the valley of $\beta$ stability, however, for some values of neutron density and temperature, branchings are open and nuclei outside the valley of stability can be produced. It can be shown that in equilibrium conditions the $s$-process distribution follows the rule $\sigma_{A} N_{A}=$ const, where $\sigma_{A}$ is the neutron capture cross section of the isotope $A$ and $N_{A}$ its abundance in number. The rule is valid locally, far from neutron magic nuclei which act as bottlenecks of the flux and produce discontinuities in the distribution. An important parameter is the neutron exposure, $\tau=\int_{0}^{t} N_{\mathrm{n}} v_{t h} d t$, where $v_{t h}$ is the thermal velocity of the gas. The neutron exposure determines the final distribution of heavy elements; for higher neutron exposures heavier elements are produced [2].

The major astrophysical site for the $s$ process has been recognised to be the deep layers of AGB stars, which are low to intermediate mass stars in a late phase of their evolution. These stars experience recurrent convective instabilities in the region between the $\mathrm{H}$ - and He-burning shells (intershell) as a consequence of thermonuclear runaways of the He-burning shell (thermal pulses, TP). At the end of a TP, the convective envelope sinks into the intershell and dredges up material to the surface (third dredge up, TDU). A large amount of ${ }^{22} \mathrm{Ne}$ is present in the convective TPs as a product of the chain

\footnotetext{
*gratefully acknowledges the support of a IPRS/MGS scholarship, maria.lugaro@maths.monash.edu.au †present address: Department of Physics \& Astronomy, University of Victoria, B.C., Canada, fherwig@mussel.phys.uvic.ca
} 
${ }^{14} \mathrm{~N}(\alpha, \gamma){ }^{18} \mathrm{~F}\left(\beta^{+} \nu\right){ }^{18} \mathrm{O}(\alpha, \gamma){ }^{22} \mathrm{Ne}$ starting on the abundant ${ }^{14} \mathrm{~N}$ from the H-burning ashes. However, the temperature in the TPs of low mass stars $\left(M \leq 4 M_{\odot}\right)$ is not high enough to significantly activate the ${ }^{22} \mathrm{Ne}(\alpha, \mathrm{n}){ }^{25} \mathrm{Mg}$ reaction. The ${ }^{13} \mathrm{C}$ neutron source is activated at lower temperatures $\left(\sim 0.8 \times 10^{8} \mathrm{~K}\right)$, however, an amount of ${ }^{13} \mathrm{C}$ higher than that present in the H-burning ashes is needed to reproduce the observed enhancements of heavy elements. To enable the formation of a region rich in ${ }^{13} \mathrm{C}$, the ${ }^{13} \mathrm{C}$ pocket, some protons from the envelope must enter the ${ }^{12} \mathrm{C}$-rich intershell. A favorable location for the occurrence of the mixing is the sharp $\mathrm{H} / \mathrm{He}$ discontinuity which is left over after TDU (see Fig. 1), however, no standard stellar models have found the penetration of protons to occur. Recently, models including time-dependent overshoot [3], motivated by hydrodynamical simulations (e.g. [4]), and models with rotation [5] have been able to create a proton-rich layer at the top of the intershell, after the end of the TDU. After less than a few thousand years ${ }^{13} \mathrm{C}$ and ${ }^{14} \mathrm{~N}$ are formed in the region. Before the end of the interpulse period all ${ }^{13} \mathrm{C}$ burns $(\alpha, \mathrm{n})$ and the region becomes enriched in $s$-processed material. At the end of the interpulse the pocket is engulfed by the following TP and is thus mixed with material from the previous TP and the ashes from the H-burning shell. Inside the TP, if the temperature at the bottom of the shell reaches $2.5 \times 10^{8} \mathrm{~K}$, the ${ }^{22} \mathrm{Ne}(\alpha, \mathrm{n})^{25} \mathrm{Mg}$ reaction is marginally activated and a second neutron flux can occur. After the TP the $s$-process-rich material from the intershell is dredged up to the envelope by the following TDU. This cycle is repeated over all TPs with TDU and the composition of the envelope throughout the AGB phase is changed by TDU and mass loss effects.

Predictions from $s$-process models have to be compared with observational constraints from spectroscopical observations of chemically peculiar stars (see e.g. [6,7]) and with laboratory measurements of isotopic anomalies in silicon carbide ( $\mathrm{SiC}$ ) grains recovered from meteorites, and believed to be originated in AGB stars [8]. Stellar models, together with Galactic Chemical Evolution models, should also reproduce the solar distribution of $s$-process elements [9]. For an extensive review on the $s$ process in AGB stars see [10].

\section{METHOD AND MODELS}

The $s$ process has been computed making use of the Torino $s$-process code (TOSP, [11]). This post-processing code calculates neutron captures on all nuclei up to $\mathrm{Pb}$ through the $\mathrm{AGB}$ phase. Since ${ }^{13} \mathrm{C}$ burns in radiative conditions, the ${ }^{13} \mathrm{C}$ pocket is simulated by specifying independent mass zones of varied extent and their ${ }^{13} \mathrm{C}$ amounts. The same ${ }^{13} \mathrm{C}$ pocket is adopted for all interpulse periods. Reaction rates for neutron production have been taken from [12] for the ${ }^{13} \mathrm{C}(\alpha, n){ }^{16} \mathrm{O}$ reaction, and from [13] for the ${ }^{22} \mathrm{Ne}(\alpha, \mathrm{n})^{25} \mathrm{Mg}$ reaction, excluding the contribution of the elusive resonance at $633 \mathrm{keV}$. In the temperature range of interest, from $\sim 2-4 \times 10^{8} \mathrm{~K}$, this rate is within a few percent of the one adopted in the NACRE compilation [14. Neutron capture rates are updated to the latest estimates $[15]$.

The post-processing models have been generated for a star of $3 M_{\odot}$ and solar metallicity with inputs such as temperatures, densities, extent of convective zones and composition of the intershell taken from three different stellar evolutionary codes: the Italian Frascati RAphson Newton Evolutionary Code (FRANEC, [16]), the Australian Mount Stromlo Stellar Structure Program (MSSSP, 17]) and the German EVOL code 18. Since the 


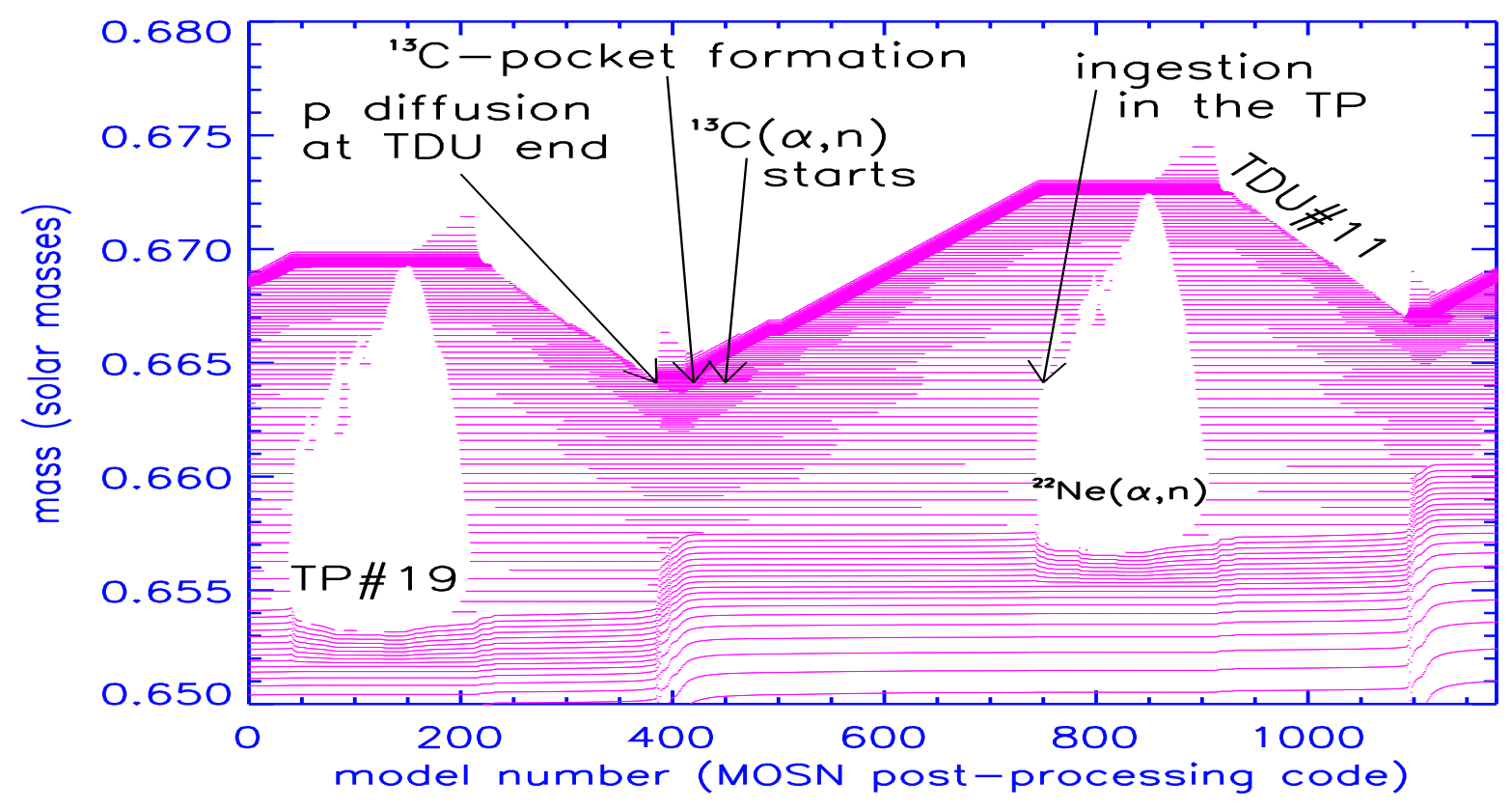

Figure 1. Structure evolution thought two TPs of the $3 M_{\odot}$ star model of solar metallicity as computed with the MSSSP and represented by the post-processing MOSN code (see §2). Convective regions are white and it is possible to recognise the convective zones of the $19^{\text {th }}$ and $20^{\text {th }} \mathrm{TPs}$, as well as the bottom of the convective envelope. The lines in the radiative regions represent the mass shells, which evolve in time. The region with the higher mesh density is the H-burning shell. The arrows give a good representation of the fact that the ${ }^{13} \mathrm{C}$ pocket is a local phenomenon involving a mass region of the order of half a tickmark on the y-axis, or less. The bottom of the convective envelope reaches $0.6643 M_{\odot}$ during the $10^{\text {th }}$ TDU and the test exercises described in $\S 2$ have been performed in the region between 0.6638 and $0.6643 M_{\odot}$.

codes are independent from one another, they contain several differences, e.g. with respect to the numerical treatment, opacities and nuclear reaction rates. The most important difference to be noted here, however, is the treatment of convective instabilities and the associated mixing. While the FRANEC code uses the Schwarzschild criterion to determine convective boundaries, the MSSSP code uses a special numerical scheme which may involve mixing of an additional stable mass shell during structure iterations. The EVOL code employs a time-dependent overshooting mechanism which leads to a deep penetration of the intershell convection zone into the $\mathrm{C} / \mathrm{O}$ core during the TP (intershell dredge up, IDU), and to the formation of a ${ }^{13} \mathrm{C}$ pocket over a range of $10^{-6}-10^{-5} M_{\odot}$. These effects depend on the value of the overshoot parameter $f$. The amount of TDU increases when moving from the FRANEC to the MSSSP to the EVOL code.

In order to study the neutron flux in the ${ }^{13} \mathrm{C}$ pocket we have made use of a code specifically designed for light-element nucleosynthesis in AGB stars: the Monash Stellar Nucleosynthesis (MOSN) code, which uses the outputs of the MSSSP code. The nuclear network contains 74 light element species, up to the iron group, and 506 reactions updated as in [19]. Neutron captures on the missing elements are modeled by a neutron sink and a 
fictional particle, $g$, is added to count the number of neutron captures occurring beyond ${ }^{62} \mathrm{Ni}$ by an invented decay, with $\lambda=1 \mathrm{~s}^{-1}:{ }^{62} \mathrm{Ni} \rightarrow{ }^{61} \mathrm{Ni}+\mathrm{g}$. Since the TOSP code does not includes proton capture reactions, the MOSN code has been useful in giving a good description of the neutron flux, particularly in regions of the pocket where the neutron poison reaction ${ }^{14} \mathrm{~N}(\mathrm{n}, \mathrm{p}){ }^{14} \mathrm{C}$ and the consequent proton recycle effect is of importance. We experimented with the MOSN code, artificially introducing some protons below the $\mathrm{H} / \mathrm{He}$ discontinuity left by the TDU to form a ${ }^{13} \mathrm{C}$ pocket. Then we let all the ${ }^{13} \mathrm{C}$ burn and checked the total neutron exposure. Since the maximum mass-shell resolution in the region of the pocket at the end of the interpulse period with the MOSN code is not smaller than $\simeq 5 \times 10^{-4} M_{\odot}$, as shown in Fig. 1, to perform each test we put a constant amount of protons, rather than a profile, over a mass of $5 \times 10^{-4} M_{\odot}$.

For the $s$-process calculations based on the FRANEC and MSSSP stellar models we used the same ${ }^{13} \mathrm{C}$ pocket profile as [11] (see their Fig. 1), which has a total mass of $5 \times 10^{-4} M_{\odot}$ and had been chosen in order to match observational constrains. The ${ }^{13} \mathrm{C}$ pocket found self-consistently with the EVOL code and used in our calculation has been computed for the $5^{\text {th }}$ interpulse period with $f=0.128$ and extents over $1.7 \times 10^{-5}$ $M_{\odot}$. To compute the resulting neutron flux we performed the same tests with the MOSN code, however, this time we artificially introduced the ${ }^{12} \mathrm{C},{ }^{13} \mathrm{C}$ and ${ }^{14} \mathrm{~N}$ found in the pocket with the EVOL code. We therefore specified the ${ }^{13} \mathrm{C}$ profile in the TOSP code to reproduce the $\tau$ calculated with MOSN.

\section{RESULTS AND DISCUSSION}

\subsection{The two neutron bursts}

The resulting ${ }^{12} \mathrm{C},{ }^{13} \mathrm{C}$ and ${ }^{14} \mathrm{~N}$ profiles in the pocket as a function of the initial $\mathrm{X}_{p}$ are presented in Fig. 2, as computed with the MOSN code. The total neutron exposure profile obtained when all ${ }^{13} \mathrm{C}$ has burnt is also plotted. Since the ${ }^{14} \mathrm{~N}(\mathrm{n}, \mathrm{p}){ }^{14} \mathrm{C}$ reaction has a relatively high cross section [20], nuclei of ${ }^{14} \mathrm{~N}$ are a strong neutron poison during the $s$ process. Some of the protons produced make more ${ }^{13} \mathrm{C}$ when recaptured by the ${ }^{12} \mathrm{C}$ (recycle effect), others destroy the ${ }^{13} \mathrm{C}$ producing more ${ }^{14} \mathrm{~N}$. As a consequence, the total neutron exposure $\tau$ grows, together with the ${ }^{13} \mathrm{C}$ profile, up to a maximum of 0.43 mbarn $^{-1}$, and then decreases when the initial abundance of ${ }^{14} \mathrm{~N}$ nuclei is comparable or higher than that of ${ }^{13} \mathrm{C}$ nuclei. This result confirms that the maximum value of $\tau$ in the pocket chosen by [11] (see their Fig. 3 and 6) is typical for stars of solar metallicity when $\mathrm{X}\left({ }^{12} \mathrm{C}\right) \sim 0.2$ in the intershell. We also made some tests changing the initial amount of

${ }^{12} \mathrm{C}$, and for different interpulse periods. As shown in Fig. 3 a nearly linear relationship exists between the final maximum value of $\tau$ in the pocket and the initial ${ }^{12} \mathrm{C}$, because the latter determines the ${ }^{13} \mathrm{C}$ and ${ }^{14} \mathrm{~N}$ profiles and how many protons will be recycled. We note that the ${ }^{12} \mathrm{C}$ in the intershell varies from pulse to pulse. This linear relationship suffers from some uncertainties. While the temperature at the $\mathrm{H} / \mathrm{He}$ interface at the end of TDU is almost constant $\left(\sim 10^{7} \mathrm{~K}\right)$ for different interpulse periods, the temperature gradient towards deeper layers is much steeper for advanced TDU. The temperature at which ${ }^{13} \mathrm{C}$ and ${ }^{14} \mathrm{~N}$ are formed in our calculations, at a mass $\simeq 3 \times 10^{-4} M_{\odot}$ below the discontinuity, is $\sim 1.5,2.2$ and $2.5 \times 10^{7} \mathrm{~K}$ after the $1^{\text {st }}$, the $10^{\text {th }}$ and the $17^{\text {th }}$ TDU respectively. The ${ }^{12} \mathrm{C},{ }^{13} \mathrm{C}$ and ${ }^{14} \mathrm{~N}$ profiles can be slightly different and, as shown in Fig. 


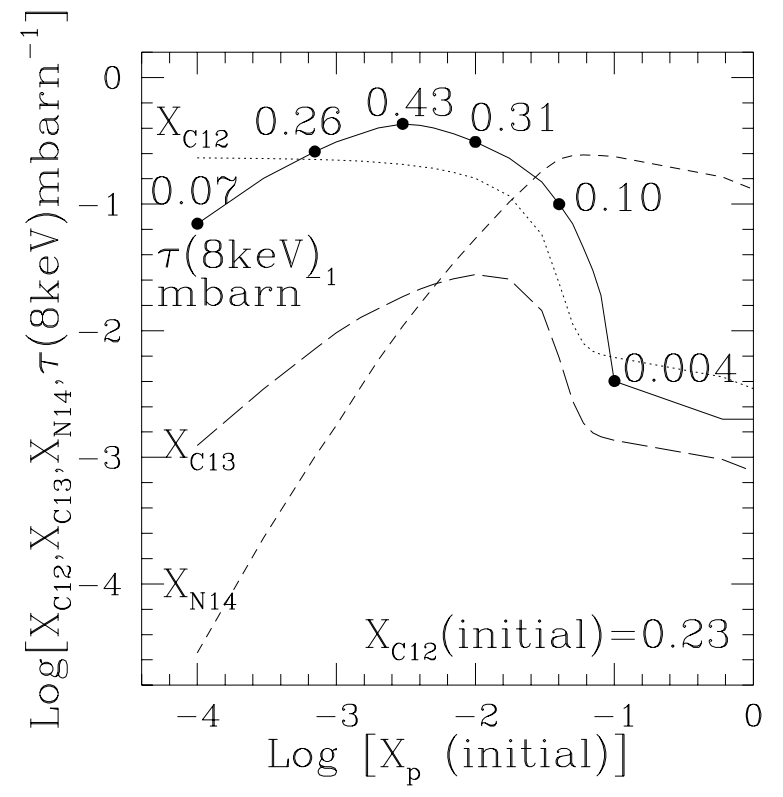

Figure 2. Resulting ${ }^{12} \mathrm{C},{ }^{13} \mathrm{C}$ and ${ }^{14} \mathrm{~N}$ profiles in the pocket as a function of the initial number of protons introduced below the $\mathrm{H} / \mathrm{He}$ discontinuity, after the end of the $10^{\text {th }}$ TDU (see Fig. 1). Also plotted is the total neutron exposure profile as computed at the end of the interpulse period after all the ${ }^{13} \mathrm{C}$ has burnt.

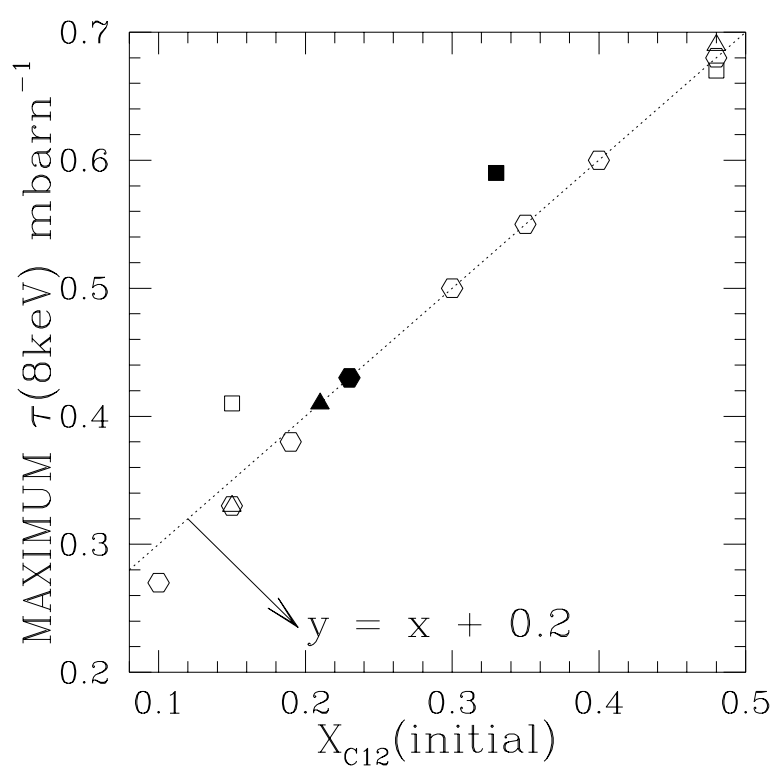

Figure 3. Maximum neutron exposure in the pocket as a function of the initial ${ }^{12} \mathrm{C}$. Squares, hexagons and triangles are results obtained for the interpulse periods following the $1^{\text {st }}, 10^{\text {th }}$ and $17^{\text {th }}$ TDU respectively. Full symbols indicate the $\mathrm{X}\left({ }^{12} \mathrm{C}\right)$ value as computed by the MSSSP at such interpulses.

3, the $\tau_{\max }$ versus ${ }^{12} \mathrm{C}$ relationship shows some spread. The smaller the extent of the pocket, the less the change of temperature gradient will effect the results. Future work includes more tests with the MOSN code, and $s$-process calculations with the TOSP code, using a different $\tau_{\max }$ for different interpulse periods.

As for the EVOL code, because of IDU, the initial mass fraction of ${ }^{12} \mathrm{C}$ is 0.48 , and the resulting abundance profiles produce a $\tau_{\max }$ of $0.88 \mathrm{mbarn}^{-1}$. The discrepancy with the maximum value of $\tau$ we found for the same initial $\mathrm{X}\left({ }^{12} \mathrm{C}\right)$ with the test profiles, computed with the MOSN code, is possibly due to a slightly lower temperature at which the pocket has been computed, because of the smaller extent in mass, and mostly to the different choice of the ${ }^{13} \mathrm{C}(\mathrm{p}, \gamma){ }^{14} \mathrm{~N}$ reaction rate. In the MOSN computation the latest rate by [21] has been adopted, rather than the previous [22], which is $30 \%$ slower.

To compare the neutron fluxes in the interpulse period and in the convective pulse, we plot in Fig. 4 the temperatures and neutron densities for the two neutron bursts, as computed by the TOSP code on the basis of the three different stellar evolution calculations. This comparison is only of qualitative character because we did not match the same TP number, core mass and envelope mass simultaneously for the three codes. However, the main finding is that the features of ${ }^{13} \mathrm{C}$ burning are fairly comparable in all cases (left panels in Fig. 4). All ${ }^{13} \mathrm{C}$ burns before the onset of the next TP because the temperature reaches $10^{8} \mathrm{~K}$ (panel $a$ ). This is a very typical situation, however, it has to be noted and 

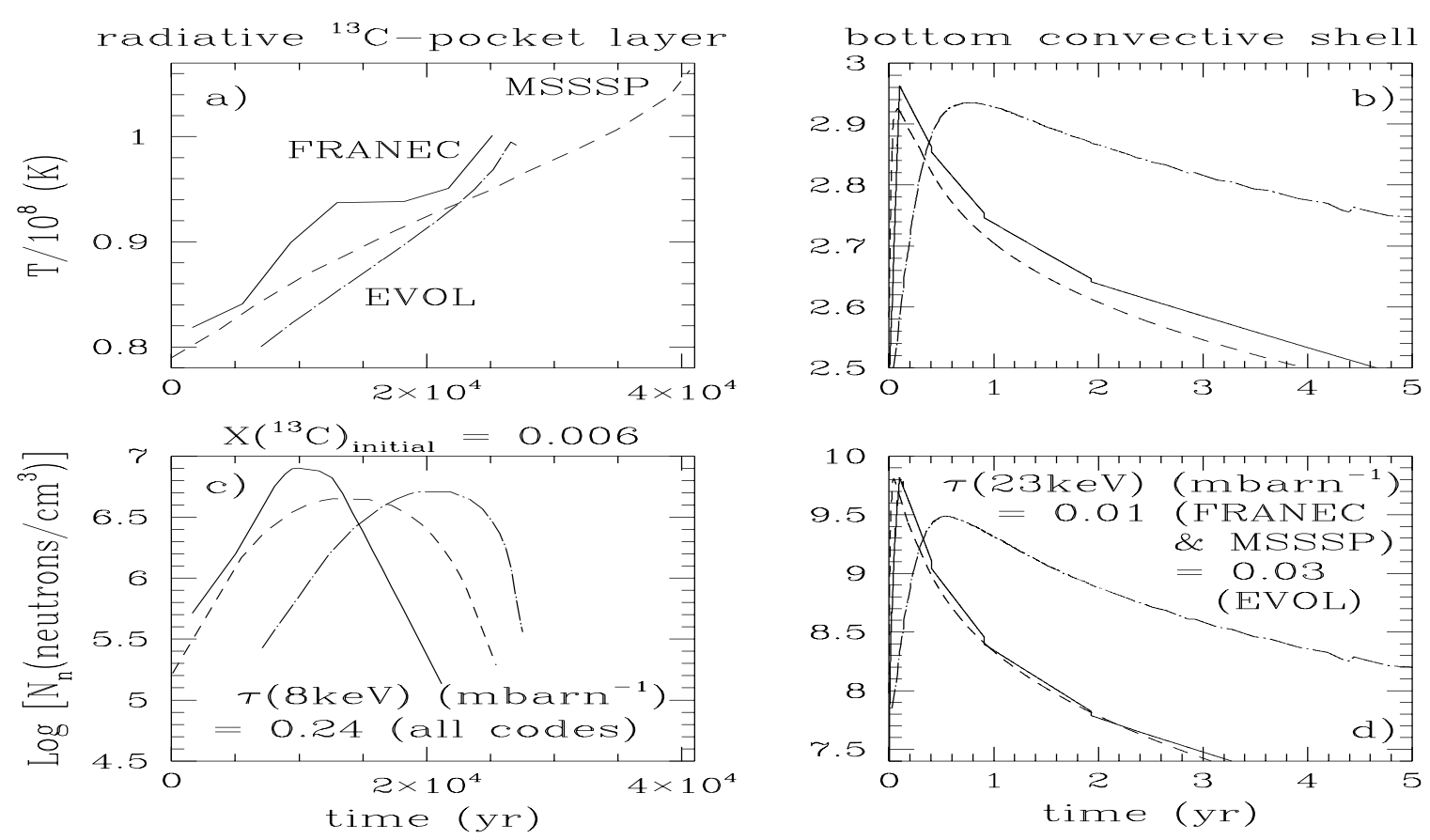

Figure 4. The two neutron bursts as described with the different codes: temperature and neutron density as computed by the TOSP code in one of the zones that forms the ${ }^{13} \mathrm{C}$ pocket for a typical interpulse period (panel $a$ and $c$ ) and at the bottom of a typical TP (panels $b$ and $d$ ). The zero points in time represent: in panels $a$ and $c$ the time from the start of the interpulse period (about 10,000 yr) when $T=0.8 \times 10^{8} \mathrm{~K}$; in panels $b$ and $d$ the time when $T_{\text {bottom }}=2.5 \times 10^{8} \mathrm{~K}$. Note the large differences in time scale, total $\tau$ and neutron-density peak between the two neutron bursts.

taken into account in future work that this might not occur during the earliest interpulse periods (see e.g. Fig. 4 of [18]). While the total neutron exposure is determined only by the initial amount of ${ }^{13} \mathrm{C}$ in any radiative layer of the pocket, the maximum neutron density can vary (panel $c$ ). However, it is so low that no branchings are activated. In the temperature range of interest the ${ }^{13} \mathrm{C}(\alpha, \mathrm{n}){ }^{16} \mathrm{O}$ rate we use is about $50-70 \%$ lower than the one adopted in [14], however, this should not make much difference in the overall result. If ${ }^{13} \mathrm{C}$ burns faster the maximum neutron density will be higher, yet still unimportant. In the TPs, the temperature profile has a narrow peak (panel $b$ ), and so has the neutron density (panel $d$ ). The neutron exposure is unimportant compared to that in the radiative layers, however, branching points are extremely sensitive to this neutron flux. Main differences show up between the FRANEC/MSSSP and the EVOL case. In the EVOL code, because of IDU, the temperature, and consequently the neutron density, stay high for a longer time and branchings are more active.

\section{2. $s$-process results}

We computed $s$-process models with the TOSP code over a total of 25 TPs with TDU for FRANEC, 18 for MSSSP and 13 for EVOL. In Table 1 the results for the envelope enrichment of $s$-process elements are compared with spectroscopic observations of AGB 
stars and their relatives. We consider the parameters 'ls', which is the average of light $s$-process elements ( $\mathrm{Y}$ and $\mathrm{Zr}$ ) and 'hs', which is the average of heavy $s$-process elements (Ba, La, Nd and $\mathrm{Sm}$ ). The ratios of these parameters with $\mathrm{Fe}$, together with the ratios $[\mathrm{hs} / \mathrm{ls}]$ and $[\mathrm{Pb} / \mathrm{Ba}]$ are good indicators of the overall distribution of heavy elements, which is determined by the main neutron exposure from the ${ }^{13} \mathrm{C}$ pocket. Predictions are given for the last TP, initial values are solar (i.e. zero). To compare with measurements in $\mathrm{SiC}$ grains we consider three isotopic ratios: ${ }^{138} \mathrm{Ba} /{ }^{136} \mathrm{Ba},{ }^{88} \mathrm{Sr} /{ }^{86} \mathrm{Sr}$ and ${ }^{96} \mathrm{Zr} /{ }^{94} \mathrm{Zr}$. The first two involve ${ }^{138} \mathrm{Ba}$ and ${ }^{88} \mathrm{Sr}$, which are neutron magic nuclei, and are thus sensitive to the neutron exposure in the ${ }^{13} \mathrm{C}$ pocket, while the third ratio involves ${ }^{96} \mathrm{Zr}$, which is produced through a branching at ${ }^{95} \mathrm{Zr}$, thus it is sensitive to the neutron density in the TPs. The ranges of prediction are given for the last phase of the evolution when the condition for $\mathrm{SiC}$ grains to form, $\mathrm{C} / \mathrm{O}>1$, is satisfied in the envelope (last 5 TPs for the FRANEC, 4 for MSSSP and 7 for EVOL).

Table 1

Results from the three computations as compared with some observational constraints.

\begin{tabular}{ccccc}
\hline parameter & measured & FRANEC & MSSSP & EVOL \\
\hline$[\mathrm{ls} / \mathrm{Fe}]^{a}$ & $0 . \rightarrow 1.5^{b}$ & 0.94 & 0.95 & 0.52 \\
{$[\mathrm{hs} / \mathrm{Fe}]^{a}$} & $0 . \rightarrow 1.5^{b}$ & 0.55 & 0.52 & 0.84 \\
{$[\mathrm{hs} / \mathrm{ls}]^{a}$} & $-0.8 \rightarrow 0.5^{b}$ & -0.40 & -0.43 & 0.35 \\
{$[\mathrm{~Pb} / \mathrm{Ba}]^{a}$} & - & -0.52 & -0.53 & 0.06 \\
$\delta\left({ }^{138} \mathrm{Ba} /{ }^{136} \mathrm{Ba}\right)^{c}$ & $\sim-250^{d}$ & $-289 \rightarrow-272$ & $-331 \rightarrow-332$ & $398 \rightarrow 647$ \\
$\delta\left({ }^{88} \mathrm{Sr} /{ }^{86} \mathrm{Sr}\right)^{c}$ & $51 \pm 103^{e}$ & $208 \rightarrow 294$ & $20 \rightarrow 149$ & $158 \rightarrow-293$ \\
$\delta\left({ }^{96} \mathrm{Zr} /{ }^{94} \mathrm{Zr}\right)^{c}$ & $-686 \pm 187^{f}$ & $-505 \rightarrow-372$ & $-718 \rightarrow-610$ & $46 \rightarrow 1120$ \\
\hline
\end{tabular}

${ }^{a}[\mathrm{X} / \mathrm{Y}]=\log \left[(\mathrm{X} / \mathrm{Y})_{\text {meas }} /(\mathrm{X} / \mathrm{Y})_{\odot}\right] .{ }^{b}$ Range of values measured in stars of $Z \sim Z_{\odot}$ from [6], typical $2 \sigma$ error $\pm 0.25 .{ }^{c} \delta(\mathrm{x} / \mathrm{y})=\left[(\mathrm{x} / \mathrm{y})_{\text {meas }} /(\mathrm{x} / \mathrm{y})_{\odot}-1\right] \times 1000 .{ }^{d}$ Bulk SiC grains from [23]. ${ }^{e}$ Average of single grains from 24]. ${ }^{f}$ Average of single grains from [25].

Since the results from the FRANEC and MSSSP computations, which use the same ${ }^{13} \mathrm{C}$ pocket, are very similar, we can conclude that the important uncertainty in $s$-process predictions is the ${ }^{13} \mathrm{C}$ pocket, not the intrinsic differences among different codes (with no overshooting mechanism included). In the EVOL code, the neutron exposure in the pocket is higher and the production of the heavier $s$-process elements, $\mathrm{Pb}$ and the magic ${ }^{138} \mathrm{Ba}$ are favored. The $s$-process element enhancements with respect to Fe, of about an order of magnitude in AGB star envelopes, are reproduced by the models. However, due to the uncertainties of the spectroscopic measurements on the observational side, and of the ${ }^{13} \mathrm{C}$ pocket on the theoretical side, we cannot draw strong conclusions about which of the models match the observations better. The ratio ${ }^{138} \mathrm{Ba} /{ }^{136} \mathrm{Ba}$ measured in bulk $\mathrm{SiC}$ grains indicates that, on average, the maximum neutron exposure in AGB stars should not exceed $\sim 0.4 \mathrm{mbarn}^{-1}$. The ratio ${ }^{96} \mathrm{Zr} /{ }^{94} \mathrm{Zr}$ is matched when the neutron density in the TPs is not higher than about $5 \times 10^{8} \mathrm{n} / \mathrm{cm}^{3}$ for a long time, a condition not satisfied by the EVOL model (see Fig. 4), which produces ${ }^{96} \mathrm{Zr}$ in a significant amount. 
The same MSSSP $s$-process calculation was performed with a different ${ }^{13} \mathrm{C}$ profile, giving more of the total mass of the pocket to the zone with higher $\tau\left(\sim 0.4 \mathrm{mbarn}^{-1}\right)$. Previously, as in [11], greater mass was given to the region with lower $\tau\left(\sim 0.15 \mathrm{mbarn}^{-1}\right)$. We found that $[\mathrm{ls} / \mathrm{Fe}]$ and $[\mathrm{hs} / \mathrm{Fe}]$ reached 1.27 and 1.0 respectively, while $[\mathrm{hs} / \mathrm{ls}]$ and $[\mathrm{Pb} / \mathrm{Ba}] \mathrm{kept}$ to -0.27 and -0.48 . The $\delta\left({ }^{138} \mathrm{Ba} /{ }^{136} \mathrm{Ba}\right)$ did not change much: $-382 \rightarrow-368$. The

$\delta\left({ }^{88} \mathrm{Sr} /{ }^{86} \mathrm{Sr}\right)$, however, increased to $447 \rightarrow 651$, going outside the measured range and supporting the profile weighted on the lower $\tau$.

Acknowledgements. We wish to thank Roberto Gallino, John Lattanzio, Maurizio Busso and Onno Pols for discussions and inspiration, Robert Cannon for coding and Brett Hennig for help with graphics and proof reading.

\section{REFERENCES}

1. E.M. Burbidge et al., Rev. Mod. Phys. 29 (1957) 547.

2. D.D. Clayton, Principles of Stellar Evolution and Nucleosynthesis, Chicago: Univ. Chicago Press (1968) ch. 7.

3. F. Herwig et al., A\&A 324 (1997) L81.

4. H.P. Singh et al., A\&A 340 (1998) 178.

5. N. Langer et al., A\&A 346 (1999) L37.

6. M. Busso et al., in Nuclei in the Cosmos V, ed. N. Prantzos and S. Harissopulos, Editions Frontières Paris (1998) 227.

7. M. Busso et al., these proceedings.

8. R. Gallino et al., in Astrophysical Implications of the Laboratory Study of Presolar Materials, eds. T. Bernatowicz and E. Zinner, New York: AIP (1997), 117.

9. C. Travaglio et al., these proceedings.

10. M. Busso et al., ARA\&A 37 (1999) 239.

11. R. Gallino et al., ApJ 497 (1998) 388.

12. A. Denker et al., in Nuclei in the Cosmos III, ed. M. Busso et al., New York: AIP (1995) 255.

13. F. Käppeler et al., ApJ 437 (1994) 396.

14. C. Angulo et al., Nucl. Phys. A656 (1999) 3.

15. Z.Y. Bao et al., Atom. Data and Nucl. Data Tables 75 (2000) 1.

16. Straniero et al. 1997, ApJ, 478, 332.

17. C.A. Frost and J.C. Lattanzio, ApJ 473 (1996) 383.

18. F. Herwig, A\&A 360 (2000) 952.

19. M. Lugaro, in Nuclei in the Cosmos V, ed. N. Prantzos and S. Harissopulos, Editions Frontières Paris (1998) 501.

20. Yu.M. Gledenov et al., in Nuclei in the Cosmos III, ed. M. Busso et al., New York: AIP (1995) 173.

21. J.D. King et al., Nucl. phys. A 567 (1994) 354.

22. G.R. Caughlan and W.A. Fowler, Atom. Data Nucl. Data Tables 40 (1988) 283.

23. C.A. Prombo et al., ApJ 410 (1993) 393.

24. G.K. Nicolussi et al., Phys. Rev. Lett. 81 (1998) 3583.

25. G.K. Nicolussi et al., Science 277 (1997) 1281. 